\title{
Remoção de sólidos suspensos na água de irrigação utilizando mantas sintéticas não tecidas
}

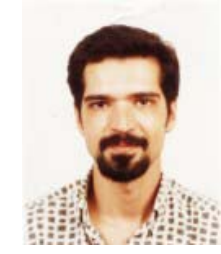

Marcos E. Scatolini \& José E. S. Paterniani²

\begin{abstract}
1 FEAGRI/UNICAMP. C.P. 215, CEP 13.825-000, Holambra, SP. Fone: (19) 3802-2316. E-mail: scatolini@dglnet.com.br (Foto) 2 DAGSOL/FEAGRI/UNICAMP. Cidade Universitária Zeferino Vaz, s/n, C.P. 6011, CEP 13083-970, Campinas, SP. Fone: (19) 3788-1019. E-mail: pater@agr.unicamp.br
\end{abstract}

Protocolo $058-30 / 4 / 2001$

\begin{abstract}
Resumo: Com vistas à eficiência de remoção de sólidos em suspensão, quando utilizados em filtros para irrigação localizada, empregaram-se três tipos diferentes de manta sintética não tecida, além de elemento de tela e de disco. Como resultado, as mantas tiveram desempenho superior, ou seja, 27 a $29 \%$ de remoção de sólidos em suspensão, enquanto os elementos de tela e disco apresentaram apenas 19 a 20\%; contudo, dentre as três mantas avaliadas não foram observadas diferenças significativas, o mesmo ocorrendo entre os elementos de tela e disco.
\end{abstract}

Palavras-chave: mantas sintéticas não tecidas, irrigação localizada, qualidade da água

\section{Removal of suspended solids in irrigation water utilizing non-woven synthetic fabrics}

\begin{abstract}
The objective of this work was to verify the efficiency of trickle irrigation filters on the removal of suspended solids using as filter elements three different types of non-woven synthetic fabrics. Also, was conducted an experiment with disk and screen elements in order to compare with the non woven synthetic fabric element. The results have demonstrated that non-woven synthetic fabrics presented better performance, removing 27 to $29 \%$ of suspended solids, while screen and disk elements presented 19 to $20 \%$ removal. No significant differences were observed among the three fabrics evaluated as well as between the screen and disk elements.
\end{abstract}

Key words: non woven synthetic fabrics, trickle irrigation, water quality

\section{INTRODUÇÃO}

Por se tratar de um método de irrigação tecnicamente eficiente e econômico, a irrigação localizada tem recebido especial atenção por parte de agricultores, irrigantes e pesquisadores. Embora este método apresente diversas vantagens em relação a outros, como economia de água, facilidade e eficiência da injeção de fertilizantes, menor exigência de mão-de-obra e redução dos riscos de contaminação de alimentos por organismos patogênicos (Paterniani et al., 1994) uma vez que a água é aplicada diretamente no solo, os principais problemas para sua implantação estão relacionados à qualidade da água a ser utilizada. Para tanto, são empregados filtros, com a finalidade de se remover partículas sólidas em suspensão presentes na água de irrigação, responsáveis pelo entupimento de emissores.

Diversos trabalhos de pesquisa e observações de campo indicam que as causas de obstrução em emissores e sistemas de filtragem podem ser divididas em três principais categorias: material em suspensão; precipitados químicos e crescimento microbiano (Nakayama, et al., 1977).
Normalmente, as obstruções são causadas pela combinação desses fatores como, por exemplo, a presença de argila e produtos de corrosão envoltos em massa biológica e cimentados com precipitados de $\mathrm{CaCO}_{3}$; no entanto, tem-se observado que os maiores problemas de obstrução são causados, em geral, pela presença de materiais em suspensão, como silte e algas (Adin \& Alon, 1986).

A eficiência ou não de diversos procedimentos e equipamentos de filtragem é função da qualidade da água utilizada na irrigação, porém uma caracterização detalhada da qualidade da água é muitas vezes de difícil realização em condições de campo e, também, pode ser prejudicada pelas variações temporais na concentração de diversos materiais orgânicos e inorgânicos.

Processos físicos de filtragem e tratamentos químicos são muitas vezes utilizados nesses sistemas, para minimizar a obstrução de emissores (Nakayama, et al., 1977; Solomon \& Keller, 1978; Oron, et al., 1980; Gilbert et al., 1981).

Os mecanismos de filtragem podem ser divididos em duas categorias: nos filtros de tela e discos, classificados como elementos de filtragem mecânica ou de superfície, cujo processo 
de filtragem é baseado no princípio segundo o qual os poros do meio filtrante são menores que o diâmetro das partículas a serem filtradas; e nos filtros granulares ou de areia, em que as partículas a serem retidas são menores que os poros do elemento filtrante, porém a retenção dessas partículas é conseguida por processos físico-químicos (Adin \& Alon, 1986).

A remoção de algas por filtros de superfície não é considerada eficiente, mas poucos experimentos têm caracterizado a capacidade de remoção desses meios filtrantes, sendo que outros mais recentes, têm demonstrado a possibilidade do uso de mantas não tecidas como elemento filtrante mais eficiente na remoção de sólidos suspensos em filtros para irrigação localizada (Paterniani \& Silva, 1996). Geralmente elas são fabricadas com fibras de polipropileno, poliamida e poliester, e possuem alta porosidade (cerca de 80 a $90 \%$, enquanto a areia apresenta porosidade em torno de $45 \%$ ) e alta superfície específica.

As características e propriedades físicas das mantas sintéticas não tecidas sugerem sua adequação para a filtração de água contendo partículas sólidas em suspensão, uma vez que não se deterioram na água e apresentam facilidade para a limpeza.

A combinação das características de porosidade, superfície específica e espessura das mantas, assim como a taxa de filtração, a qualidade da água e o sistema de filtração utilizado, são parâmetros que determinam a manta mais adequada às condições de filtração (Paterniani, 1991).

Silva (1996) realizou diversos ensaios experimentais simulando contaminação na água por sólidos suspensos, utilizando areia em concentrações de 70 a $300 \mathrm{mg} \mathrm{L}^{-1}$, observando que filtros de manta removeram de 15 a $25 \%$ dos sólidos suspensos, enquanto os elementos de tela e disco removeram de 2 a $10 \%$.

No presente trabalho avaliou-se e se comparou a eficiência de remoção de partículas sólidas em suspensão presentes na água de irrigação, por mantas sintéticas não tecidas, elementos de disco e de tela.

\section{MATERIAL E MÉTODOS}

Os ensaios foram realizados em uma propriedade agrícola, na qual foi captada água de um reservatório superficial, cuja água é utilizada para irrigação de vasos com mudas de flores. Neste reservatório foi instalado um conjunto de bombeamento, que alimentou 5 filtros plásticos de 1" colocados em paralelo e, portanto, sob as mesmas condições de perda de carga. A vazão média através de cada filtro foi de $2,4 \mathrm{~m}^{3} \mathrm{~h}^{-1}$, porém o tempo de ensaio variou em função da concentração de sólidos em suspensão. Foram instalados, na saída dos filtros, reguladores de pressão de $106 \mathrm{kPa}$, a fim de possibilitar que a vazão após os mesmos não fosse afetada pela obstrução dos elementos filtrantes. Desta maneira, haveria a possibilidade de instalação de linhas de gotejadores trabalhando sob condições semelhantes, embora testes com gotejadores não tenham sido objetivo da presente pesquisa. Os 5 elementos filtrantes utilizados foram tela de nylon 120 mesh, discos 120 mesh, manta M2, manta M3 e manta M4 (Tabela1).
Tabela 1. Característica físicas das mantas sintéticas não tecidas

\begin{tabular}{lccc}
\multicolumn{1}{c}{ Mantas } & M2 & M3 & M4 \\
\multicolumn{1}{c}{ COR } & Cinza & Preta & Cinza N \\
\hline Massa específica do fio $-\rho_{\mathrm{f}}\left(\mathrm{g} \mathrm{cm}^{-3}\right)$ & 1,1237 & 0,9835 & - \\
Massa específica da manta $-\rho_{\mathrm{f}}\left(\mathrm{g} \mathrm{cm}^{-3}\right)$ & 0,1235 & 0,1151 & 0,10 \\
Porosidade $-\varepsilon_{\mathrm{o}}(\%)$ & 89,01 & 88,29 & - \\
Diâmetro médio do fio $-\mathrm{d}_{\mathrm{f}}(\mu \mathrm{m})$ & 42,43 & 45,09 & - \\
Superfície específica $-\mathrm{S}_{\mathrm{o}}\left(\mathrm{m}^{2} \mathrm{~m}^{-3}\right)$ & 10360 & 10388 & - \\
Permeabilidade $\left(\mathrm{mm} \mathrm{s}^{-1}\right)$ & 7,01 & 7,16 & 5,0 \\
Espessura $(\mathrm{mm})$ & - & - & 3,8 \\
Abertura de filtragem $(\mu \mathrm{m})$ & - & - & 150 \\
\hline
\end{tabular}

A qualidade da água em estudo apresentou concentração de sólidos em suspensão, que variou de 1,13 a $14,75 \mathrm{mg} \mathrm{L}^{-1}$, com média de $5,59 \mathrm{mg} \mathrm{L}^{-1}$. Embora não se tenha determinado outros parâmetros qualitativos desta água, pode-se pelos dados disponíveis, classificar a água em estudo com baixo potencial de obstrução de gotejadores, segundo (Nakayama, 1982).

Realizaram-se 27 ensaios de filtragem para cada um dos 5 tratamentos (tela, discos manta M2, manta M3 e manta M4). Durante cada ensaio coletaram-se 4 amostras de água do afluente e do efluente, para a determinação da concentração média de sólidos em suspensão e, conseqüentemente, a porcentagem de remoção de cada elemento filtrante (Eq. 1).

$$
\mathrm{REM}=100[1-(\mathrm{Efl} / \mathrm{Afl})]
$$

donde:

REM - remoção de sólidos em suspensão, \%

Efl - concentração de sólidos em suspensão no efluente, $m g \mathrm{~L}^{-1}$

Afl - concentração de sólidos em suspensão no afluente, $\mathrm{mg} \mathrm{L} \mathrm{L}^{-1}$

Os ensaios de filtração foram monitorados continuamente por um Datalogger, cujos dados de perda de carga ficaram registrados ao longo do tempo e, posteriormente, transportados para um microcomputador, através de um sistema de aquisição de dados, para confecção do gráfico (Figura 1).

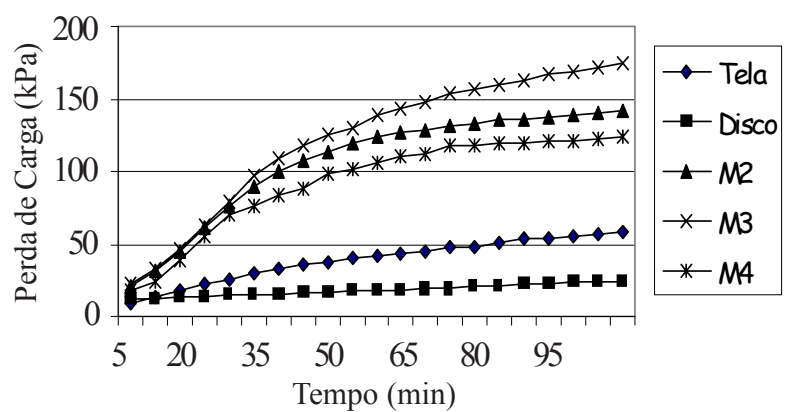

Figura 1. Perda de carga média dos elementos filtrantes tela, disco e mantas não tecidas M2, M3 e M4 medida ao longo dos ensaios

A determinação da concentração de sólidos em suspensão totais das amostras de água coletada, empregada no cálculo da porcentagem de remoção através da Eq. 1, seguiu rigorosamente a metodologia descrita no Standard Methods for Examination of Water and Wastewater, (APHA 1995). 
Os dados, em porcentagem, foram transformados segundo $\mathrm{o}$ arco seno da raiz de $\mathrm{x}$, para garantia da normalidade, premissa básica para as análises de variância, segundo procedimento indicado por Steel \& Torrie, 1980. A partir desses dados, utilizou-se o teste de Duncan para comparação das médias.

\section{RESULTADOS E DISCUSSÃO}

Os resultados de remoção de sólidos suspensos indicaram coeficiente de variação elevado devido, talvez, à variação nas dimensões das partículas presentes na água, que proporcionaram maior ou menor porcentagem de remoção (Tabela 2); no entanto, a análise estatística dos dados ajustados demonstrou que a porcentagem média de remoção dos elementos filtrantes foi de $19 \%$ para o elemento de discos, $20 \%$ para o elemento de tela, $27 \%$ para a manta M2 e M3 e $29 \%$ para a manta M4. Verificou-se que a remoção da tela e do disco não diferiu significativamente e que as três mantas diferiram significativamente da tela e do disco, mas não entre elas (Tabela 3). Estes dados caracterizam a maior eficiência das três mantas não tecidas na remoção de sólidos suspensos presentes na água de irrigação avaliada, mesmo para a quantidade de sólidos suspensos considerada baixa de acordo com a escala proposta por Nakayama (1982).

Embora a determinação com precisão do potencial de obstrução dos emissores em função da qualidade da água seja

Tabela 2. Concentrações de sólidos em suspensão e remoção nos diferentes tipos de filtro para os 27 ensaios realizados

\begin{tabular}{|c|c|c|c|c|c|c|}
\hline \multirow{2}{*}{$\begin{array}{l}\text { Número } \\
\text { do Ensaio }\end{array}$} & \multirow{2}{*}{$\begin{array}{l}\mathrm{CMA}^{*} \\
\mathrm{Mg} \mathrm{L}^{-1}\end{array}$} & \multicolumn{5}{|c|}{ Remoção de Sólidos em Suspensão (\%) } \\
\hline & & Tela & Disco & M4 & M3 & M2 \\
\hline 1 & 5,13 & 8,9 & 7,1 & 23,8 & 16,3 & 20,3 \\
\hline 2 & 3,29 & 10,9 & 10,9 & 21,5 & 17,3 & 23,3 \\
\hline 3 & 3,92 & 14,9 & 20,2 & 18,1 & 18,5 & 21,3 \\
\hline 4 & 6,58 & 5,8 & 4,8 & 22,2 & 18,3 & 27,8 \\
\hline 5 & 6,58 & 11,4 & 7,6 & 14,6 & 15,2 & 15,8 \\
\hline 6 & 4,17 & 13,1 & 12,0 & 14,7 & 17,0 & 16,6 \\
\hline 7 & 3,17 & 15,8 & 21,1 & 36,8 & 23,7 & 26,3 \\
\hline 8 & 10,04 & 9,3 & 12,3 & 12,0 & 10,4 & 11,3 \\
\hline 9 & 6,12 & 7,5 & 7,5 & 11,6 & 12,9 & 10,6 \\
\hline 10 & 5,33 & 9,4 & 6,3 & 25,0 & 14,8 & 15,2 \\
\hline 11 & 1,13 & 23,0 & 11,1 & 25,9 & 23,0 & 18,5 \\
\hline 12 & 3,96 & 16,8 & 13,7 & 22,1 & 22,1 & 20,0 \\
\hline 13 & 4,63 & 15,3 & 12,6 & 23,4 & 14,4 & 15,3 \\
\hline 14 & 1,38 & 27,3 & 30,3 & 35,4 & 37,0 & 27,3 \\
\hline 15 & 8,17 & 14,3 & 24,5 & 50,0 & 42,9 & 48,0 \\
\hline 16 & 4,92 & 10,2 & 11,9 & 30,5 & 30,5 & 30,5 \\
\hline 17 & 2,46 & 18,6 & 16,9 & 39,0 & 33,9 & 32,2 \\
\hline 18 & 14,75 & 13,0 & 14,7 & 44,1 & 33,3 & 32,2 \\
\hline 19 & 11,42 & 3,6 & 5,1 & 25,5 & 24,8 & 23,4 \\
\hline 20 & 5,71 & 8,8 & 5,6 & 16,8 & 13,1 & 16,1 \\
\hline 21 & 4,50 & 22,2 & 11,1 & 27,8 & 27,8 & 22,2 \\
\hline 22 & 4,67 & 10,7 & 7,1 & 21,4 & 14,3 & 16,1 \\
\hline 23 & 8,75 & 10,5 & 9,5 & 26,7 & 21,9 & 23,8 \\
\hline 24 & 7,33 & 8,0 & 5,7 & 13,6 & 13,6 & 13,6 \\
\hline 25 & 3,75 & 11,8 & 8,1 & 24,4 & 22,2 & 22,2 \\
\hline 26 & 3,58 & 9,3 & 7,0 & 16,3 & 14,0 & 18,6 \\
\hline 27 & 5,42 & 7,7 & 4,6 & 16,0 & 10,3 & 14,6 \\
\hline Médias & 5,29 & 12,5 & 11,5 & 24,4 & 20,9 & 21,6 \\
\hline $\begin{array}{l}\text { Desvio } \\
\text { Padrão }\end{array}$ & 3,02 & 5,4 & 6,4 & 9,8 & 8,6 & 8,0 \\
\hline CV (\%) & 54 & 44 & 56 & 40 & 41 & 37 \\
\hline
\end{tabular}

Tabela 3. Resumo da análise da variação e média da remoção de sólidos suspensos observada para diferentes tratamentos

\begin{tabular}{lcccc}
\hline $\begin{array}{l}\text { Fonte } \\
\text { de Variação }\end{array}$ & G.L. & S.Q. & Q.M. ${ }^{1}$ & F \\
\hline Tratamento & 4 & 2197,3794 & 549,3448 & 17,6805 \\
Resíduo & 130 & 4039,1934 & 31,0707 & \\
Total & 134 & 6236,5728 & & \\
Média & 24,5844 & & & \\
C.V. (\%) & 26,67 & & & \\
Média $^{2}$ & & & & \\
Manta M4 & 29,227743 & & $(23,841965)$ & $\mathrm{a}$ \\
Manta M2 & 27,379892 & & $(21,149718)$ & $\mathrm{a}$ \\
Manta M3 & 26,790359 & & $(20,315519)$ & $\mathrm{a}$ \\
Tela & 20,324263 & & $(21,064010)$ & $\mathrm{b}$ \\
Disco & 19,199793 & & $(10,815106)$ & $\mathrm{b}$ \\
\hline
\end{tabular}

${ }^{1}$ Com médias transformadas em $\operatorname{arcsen} \sqrt{\mathrm{x}}$

${ }^{2}$ Valores entre parênteses indicam os valores originais. Médias seguidas por letras distintas diferem a 0,01 de probabilidade, pelo teste de Duncan

muito difícil, a escala proposta por Nakayama (1982) para identificação de situações problemáticas, classificando-a em baixa, moderada e severa, de acordo com a concentração de substâncias físicas, químicas e biológicas presentes na água classifica como baixo o risco de obstrução de emissores para concentração de sólidos em suspensão inferior a $50 \mathrm{mg} \mathrm{L}^{-1}$, porém se observou, durante o ensaio que, mesmo para concentração média de sólidos em suspensão de $5,6 \mathrm{mg} \mathrm{L}^{-1}$, o tempo de filtração não foi superior a $4 \mathrm{~h}$, devido ao crescimento da perda de carga, com razoável quantidade de sólidos retidos nos meios filtrantes. A determinação da quantidade de sólidos suspensos retidos no meio filtrante foi feita multiplicando a quantidade de sólidos suspensos presentes na água de irrigação pelo vazão média que passou pelos meios filtrantes, pelo tempo de funcionamento médio dos ensaios e pela porcentagem média de remoção dos elementos filtrantes. Como a concentração média de sólidos suspensos foi de $5,6 \mathrm{mg} \mathrm{L}^{-1}$, o tempo médio de duração dos ensaios foi de $4 \mathrm{~h}$ e a vazão média através dos meios filtrantes foi de $2400 \mathrm{~L} \mathrm{~h}^{-1}$, quantidade de impurezas retidas no meio filtrante seria aproximadamente de $54 \mathrm{~g}$ para o período referido, numa superfície de $173 \mathrm{~cm}^{2}$, no caso de $100 \%$ de remoção. No presente caso, como a porcentagem de remoção variou entre 19,2 e 29,2\%, determinou-se que a quantidade média de sólidos em suspensão retido nos elementos filtrantes, foi de 10,4 g para os elementos de tela e disco e de $15,8 \mathrm{~g}$ para o elemento de tela $\mathrm{M} 4$, ou seja, a manta reteve $52 \%$ a mais que os elementos de tela e disco, o que também explica o crescimento mais acelerado da perda de carga nas mantas sintéticas não tecidas, uma vez que todos os elementos filtrantes têm similar perda de carga quando limpos, o que pode ser observado na Figura 1 no início do processo de filtragem. Considerando-se que a ação de superfície nos filtros de manta é predominante na retenção de impurezas sólidas, aumentando a superfície do meio filtrante, supõe-se ser possível obter carreiras de filtração de maior duração compensando, assim, a perda de carga mais elevada encontrada nas mantas não tecidas.

Embora uma análise econômica não seja objetivo principal do presente projeto de pesquisa, mesmo porque é difícil e pouco confiável estabelecer-se uma comparação em níveis econômicos e de custos entre produtos fabricados em escala comercial com um que não é fabricado nessas condições, uma análise criteriosa 
deveria levar em conta os custos indiretos de fabricação (desenvolvimento do produto, alterações na linha de montagem etc) e não simplesmente o custo dos componentes do produto.

Contudo, esta seria uma importante informação para o usuário, ou mesmo para pretensões de fabricação e/ou comercialização por parte de algum fabricante de filtro. O que se pode afirmar, porém, é que os filtros de tela e discos existentes no mercado são, na sua grande maioria, se não na totalidade, de fabricação estrangeira, susceptíveis, assim, às constantes variações cambiais que ocorrem com certa freqüência no Brasil. A possibilidade de se ter no mercado um produto nacional com qualidade superior ou igual ao importado e a um custo menor e estável, seria sem dúvida bem aceito pelos usuários. A fabricação de filtros de discos e de tela no Brasil seria viável, mas certamente, esses filtros seriam mais caros que os de mantas não tecidas, propostos nesta pesquisa, uma vez que as telas e principalmente os discos, demonstram necessitarem de um controle de qualidade elevado, em vista, de suas características específicas (abertura de poros, espessuras e ranhuras micrométricas, além de encaixes perfeitos), ao passo que na fabricação de filtros com o uso de mantas, ter-se-ia plenas condições de terceirizá-los, visto que o parque industrial brasileiro dos não tecidos vem crescendo de forma bastante intensa (www.abint.org.br).

\section{CONCLUSÕES}

As principais conclusões extraídas do presente trabalho foram:

1. As mantas sintéticas utilizadas como elemento filtrante, operaram satisfatoriamente, mostrando funcionalidade e resistência.

2. As mantas sintéticas foram significativamente mais eficientes que os elementos de tela e disco, removendo maior quantidade de sólidos em suspensão quando empregadas em filtros de 1" de diâmetro.

3. A evolução da perda de carga nas mantas foi mais rápida que nos elementos de tela e discos, devido principalmente ao maior acúmulo de resíduos em sua superfície, decorrência direta de sua maior eficiência.

\section{AGRADECIMENTOS}

Os autores agradecem à FAPESP, pelas bolsas concedidas e auxílio à pesquisa. (Processos: 97/6570-9, 97/05171-3, 97/01704-4) .

\section{LITERATURA CITADA}

Adin, A.; Alon, G. Mechanisms and process parameters of filter screens. Journal of Irrigation and Drainage Engineering. New York. v.112, n.4, p.293-304, 1986.

APHA - American Public Health Association. Standard methods for the examination of water and wastewater. 19. ed., Washington D.C.: APHA/AWWA/WPCF. 1995. 798p.

Gilbert, R.G.; Nakayama, F.S.; Bucks, D.A.; French, O.F.; Adamson, K.C.; Johnson, R.M. Trickle Irrigation: Predominant bacteria in treated Colorado river water and biologically clogged emitters. Transactions of the ASAE, St. Joseph, v.24, n.1, p.77-80. 1981

Nakayama, F.S. Water analysis and treatment techniques for control emitter plugging. In: Proceedings Irrigation Association Conference, Portland, Oregon, 1982.

Nakayama, F.S.; Bucks, D.A.; French, O.F. Reclaiming partially clogged trickle emitters. Transactions of the ASAE, St. Joseph, v.20, n.2, p. 278-280, 1977.

Oron, G.; Shelef, G.; Zur, B. Stormwater and reclaimed effluent in trickle irrigation. Journal of the Irrigation and Drainage Division, New York, v.106, n.4, p.299-310, 1980.

Paterniani, J.E.S. Utilização de mantas sintéticas não tecidas na filtração lenta em areia de águas de abastecimento. São Carlos: USP, 1991, 245p. Tese Doutorado

Paterniani, J.E.S.; Matsura, E.E.; Roston, D.M.; Paula Jr., D.R.; Testezlaf, R.; Ribeiro, T.A.P. Diagnóstico da qualidade da água de irrigação em propriedades produtoras de hortaliças da região de Campinas. In: Congresso Brasileiro de Engenharia Agrícola 23. 1994. Campinas. Anais... Campinas: SBEA, 1994. p.20

Paterniani, J.E.S.; Silva, L.B. Comparação entre diferentes meios filtrantes de filtros para irrigação localizada. In: Congresso Argentino, 4 e Congresso Internacional de Ingenieria Rural, 2, Anais... 1996, Neuquen: Editorial Facultad de Agronomia de Buenos Aires, 1996. p.397-401.

Silva, L.B. Utilização de mantas sintéticas não tecidas na filtração sob pressão. Campinas: UNICAMP, 1996, 103p. Dissertação Mestrado

Solomon, K.H.; Keller, J. Trickle irrigation uniformity and efficiency. Journal of the Irrigation and Drainage Division, New York, v.104, n.3, p.293-306, 1978.

Steel, R.G.; Torrie, J.K. Principles and procedures of statistics: A biometrical approach, 2 ed. Tokyo: Mc Graw-Hill, 1980, 663p. WWW (World Wide Web). www.abint.org.br, 2000. 